\title{
Identification of microsatellite loci, gene ontology and functional gene annotations in Indian salmon (Eleutheronema tetradactylum) through next- generation sequencing technology using illumina platform
}

\author{
Shihab Ismail*, N. Vineesh, Reynold Peter, P. Vijayagopal, A. Gopalakrishnan \\ ICAR Central Marine Fisheries Research Institute, Post Box No: 1603, Ernakulam North P.O., Kochi, Kerala, 682018, India
}

\section{A R T I C L E I N F O}

\section{Keywords:}

Microsatellites

Indian salmon

Population genetics

Genetic stocks

Gene ontology

\begin{abstract}
A B S T R A C T
Whole genome sequencing was performed on three samples of four finger threadfin Eleutheronema tetradactylum $\left(\mathrm{KET}_{25}, \mathrm{KET}_{29}\right.$ and $\left.\mathrm{KET}_{30}\right)$ using illumina NextSeq500 platform using $2 \times 150$ bp chemistry. 8,390,317, $7,085,775$ and $8,461,589$ high quality reads were obtained after trimming low quality reads and adapter sequence. These high quality reads obtained were used for de novo assembly and obtained a number of scaffolds. From these scaffolds of vast sequenced data, we were able to identify 60246, 46107 and 60907 Simple Sequence Repeats (SSR) markers in $\mathrm{KET}_{25}, \mathrm{KET}_{29}$ and $\mathrm{KET}_{30}$ respectively, which will be useful in population genetic analysis and other diversity studies in Indian salmon. The gene prediction on assembled scaffolds predicted 31,943 genes for $\mathrm{KET}_{25} ; 26,487$ genes for $\mathrm{KET}_{29}$ and 31,654 genes for $\mathrm{KET}_{30}$ with average gene size of 458bp, 424bp and 459bp respectively. A total of 30,209, 25,107 and 29,943 genes were annotated against the NCBI Nr database for the samples respectively. E. tetradactylum is a commercially important fish species for many countries. This is the first report on the identification of genomic SSR markers in E. tetradactylum using NGS technology. This study provides an insight of baseline knowledge of the genome sequence of Indian salmon for future studies.
\end{abstract}

\section{Introduction}

NGS technologies are making a notable footprint on many areas of biology, including the genetic diversity in populations [1]. NGS is not only a simple genome sequencing method, but have greatly beneficial to the fields of biology, epidemiology, evolutionary biology, phylogenetics, comparative genomics, microbial diversity, DNA marker discovery and studies of gene function and expression [2]. NGS technologies have radically changed the way genetic sequence data are generated and have accelerated a revolution in biological research [3]. Recent developments in NGS technologies have sophisticated the rapid and economical discovery of molecular markers from non-model organisms [4]. Recent developments in sequencing technology, short read sequencers (90-400 bp), such as Illumina and Ion Torrent, are starting to be more frequently used for the generation of large NGS data sets, with affordable price range [5]. NGS has enabled the rapid and cost effective genetic marker discovery, including microsatellites and SNPs and has empowered the massive increase in the number of sequence attained per sequencing effort and also led to the development of highoutput genotyping by sequencing [6]; Davey et al., 2011 and [7].
The vast and huge sequencing data generated through NGS technology and whole genome sequencing enables the development of molecular markers for population genetics, molecular systematics, evolutionary developmental biology and gene mapping studies. Population genetics rely essentially on two types of genetic markers; microsatellite markers or short tandem repeats (STRs) and single-nucleotide polymorphisms (SNPs). Microsatellites have been used widely since the late eighties for applications such as parentage analysis, population genetic structure and conservation genetics because of their high level of polymorphism (allelic richness), higher mutation rate, relatively small size and higher statistical power per locus (rapid analysis protocol) $[8,9]$. Microsatellites, also known as SSRs, are tandem repeated motifs of 1-6 bases and serve as the most important molecular markers in population and conservation genetics, molecular epidemiology and pathology and gene mapping. Majority of population genetic studies in marine fisheries have employed microsatellites, due to its high mutation rate which may results in extremely high level of variation in marine fish [10]. The development of suitable molecular markers would favour studies of wild population structure that will finally result in improved broodstock selection [11].

\footnotetext{
* Corresponding author.

E-mail address: shihabismail51@gmail.com (S. Ismail).
} 
Microsatellites generally require species-specific marker development that can be expensive and laborious and limited by the difficulties of de novo development in species without any prior genomic information $[12,13]$. Other problems analogous with microsatellites include poor level of inter-laboratory calibration with genotype based on fragment size, fragment size-homoplasy and laborious genotyping [14]. Many of these issues associated with microsatellite based population studies could be eliminated using NGS based microsatellite approach leading to faster and cheaper genotyping in large-scale population genetic studies [15]. The use of microsatellites will only outweigh the use of SNPs if microsatellites can be generated from NGS platforms and used with programs such as MEGASAT [16] for genotype calling.

Eleutheronema tetradactylum or four finger threadfin commonly known as blue threadfin also called Indian salmon, is a marine protandrous hermaphrodite species [17] belong to the family polynemidae, and they are distributed in tropical and subtropical waters throughout the world. They are generally found in coastal marine waters, estuaries or rivers in the tropics [18]. They mainly feed on small fishes, prawns, shrimps and mysids and adult fish prey on other fishes [18]. In Western Australia they are considered fully or over-exploited [19,20]. Stock structure analysis of blue threadfin in Australia using parasite and tagrecapture data [21] and by comparing the life history parameters [22] suggests the possibility of separate sub-stocks. There is limited information about the genetic stock structure of this commercially important fish species from Indian waters. As E. tetradactylum is commercially important species of the region, it is very essential to utilize the fishery in a sustainable manner.

Here we present the microsatellite identification, gene ontology and functional gene annotations for a hermaphroditic species Indian salmon through NGS technology using Illumina platform. The microsatellite markers identified through this NGS technology herein offer important genetic resources for the assessment, understanding and conservation of this hermaphroditic species and facilitate future work on the other related species.

\section{Materials and methods}

\subsection{Sampling and DNA isolation}

Fresh samples of 6 Indian salmon were collected from commercial fishing operations from Indian waters. Each fish was measured for Total Length (TL) to the $1.0 \mathrm{~mm}$ below and total body weight to the nearest $0.01 \mathrm{~g}$. The body cavity was dissected out to assess sex and maturity. A piece of tissue was excised from caudal peduncle of each specimen and stored at $4{ }^{\circ} \mathrm{C}$ in absolute ethanol. Total genomic DNA was extracted from $10 \mathrm{mg}$ of tissue from each fish using a chloroform/isoamyl alcohol protocol. Extracted DNA was quantified on a NanoDrop ND-1000 spectrophotometer and was stored at $-20{ }^{\circ} \mathrm{C}$ until further use. Isolated genomic DNA was sent to the Eurofins Genomics India Private limited (Bangalore, India) for library preparation and sequencing.

\subsection{Qualitative and quantitative analysis of isolated gDNA}

Quality of the fish muscle gDNA was checked on NanoDrop and $2 \mu \mathrm{l}$ of DNA was resolved on $0.8 \%$ Agarose gel at $120 \mathrm{~V}$ for approximately $60 \mathrm{~min}$ or until the samples reached 3/4th of the gel. $1 \mu \mathrm{l}$ of sample was loaded in NanoDrop for determining A260/280 ratio and also quantified by Qubit 3.0. All the DNA samples passed the QC and of these three samples $\left(\mathrm{KET}_{25} ; \mathrm{KET}_{29}\right.$ and $\left.\mathrm{KET}_{30}\right)$ were randomly selected for pairedend sequencing library preparation.

\subsection{Preparation of $2 \times 150$ NextSeq500 libraries}

The paired-end sequencing libraries were prepared from the QC passed gDNA samples $\left(\mathrm{KET}_{25} ; \mathrm{KET}_{29}\right.$ and $\left.\mathrm{KET}_{30}\right)$ using TruSeq Nano DNA library Prep Kit (Illumina, San Diego, CA). 200 ng of Genomic
Table 1

Summary of gene Assembly in E. tetradactylum.

\begin{tabular}{llll}
\hline Description & KET25 & KET29 & KET30 \\
\hline Number of scaffolds & 289,461 & 280,260 & 289,650 \\
Total size of assembly & $301,704,044$ & $263,802,638$ & $306,253,351$ \\
Average size of scaffolds & 1042 & 941 & 1057 \\
Scaffold N50 & 1157 & 1001 & 1180 \\
Maximum size of scaffold & 16,681 & 17,051 & 16,622 \\
Minimum size of scaffold & 500 & 500 & 500 \\
\hline
\end{tabular}

Table 2

Gene statistics in three samples of $E$. tetradactylum.

\begin{tabular}{llll}
\hline Description & KET25 & KET29 & KET30 \\
\hline Number of genes & 31,943 & 26,487 & 31,654 \\
Average gene length & 458 & 424 & 459 \\
Maximum gene length & 5070 & 5493 & 4848 \\
Minimum gene length & 201 & 201 & 201 \\
\hline
\end{tabular}

DNA from the three samples was fragmented by Covaris M220 (Covaris, Woburn, MA) to generate a mean fragment distribution of $400 \mathrm{bp}$. The focussed ultrasonic shearing using Covaris generates dsDNA fragments with $3^{\prime}$ to $5^{\prime}$ overhangs. The fragments were then subjected to endrepair. This process converts the overhangs resulting from fragmentation in to blunt ends using End Repair Mix. The $3^{\prime}$ to $5^{\prime}$ exonuclease activity of this mix removes the $3^{\prime}$ overhangs and the $5^{\prime}$ to $3^{\prime}$ polymerase activity fills in the $5^{\prime}$ overhangs followed by adapter ligation to the fragments. This strategy ensures a low rate of chimera formation. The ligated products were size selected using AMPure XP beads (NEB, Ipswich, MA). The size selected product range between 478bp to $492 \mathrm{bp}$ was PCR amplified with the index primer. Indexing adapters were ligated to the ends of the DNA fragments, preparing them for hybridization on to a flow cell.

\subsection{Quality check $(Q C)$ of library, cluster generation and sequencing}

The PCR amplified libraries were analysed on Tape Station 4200 (Agilent Technologies) using High sensitivity D1000 Screen Tape assay kit as per manufacturer instructions. After obtaining the Qubit concentration for the libraries and the mean peak size from Agilent Tape Station profile, the PE illumina libraries were loaded onto NextSeq 500 for cluster generation and sequencing. Paired-End sequencing allows the template fragments to be sequenced in both the forward and reverse directions on NextSeq 500. The kit reagents were used for binding of samples to complementary adapter oligos on paired-end flow cell. The adapters were designed to allow selective cleavage of the forward strands after re-synthesis of the reverse strand during sequencing. The copied reverse strand will then use to sequence from the opposite end of the fragments.

\subsection{Statistical analysis}

The sequenced raw data was processed to obtain high quality clean reads using Trimmomatic v0.35 to remove adapter sequences, ambiguous reads (reads with unknown nucleotides "N" larger than 50\%), and low-quality sequences (reads with more than $10 \%$ quality threshold $(\mathrm{QV})<20$ phred score). A minimum length of 75 nucleotide after trimming was applied. After removing the adapter and low quality sequences from the raw data, 8,390,317 $(2 \times 150 \mathrm{bp}), 7,085,775$ $(2 \times 150 \mathrm{bp})$ and $8,461,589(2 \times 150 \mathrm{bp})$ high quality reads were retained for $\mathrm{KET}_{25}, \mathrm{KET}_{29}$ and $\mathrm{KET}_{30}$ samples respectively. This high quality $(\mathrm{QV}>20)$, paired-end reads were used for de novo assembly of all the samples. 


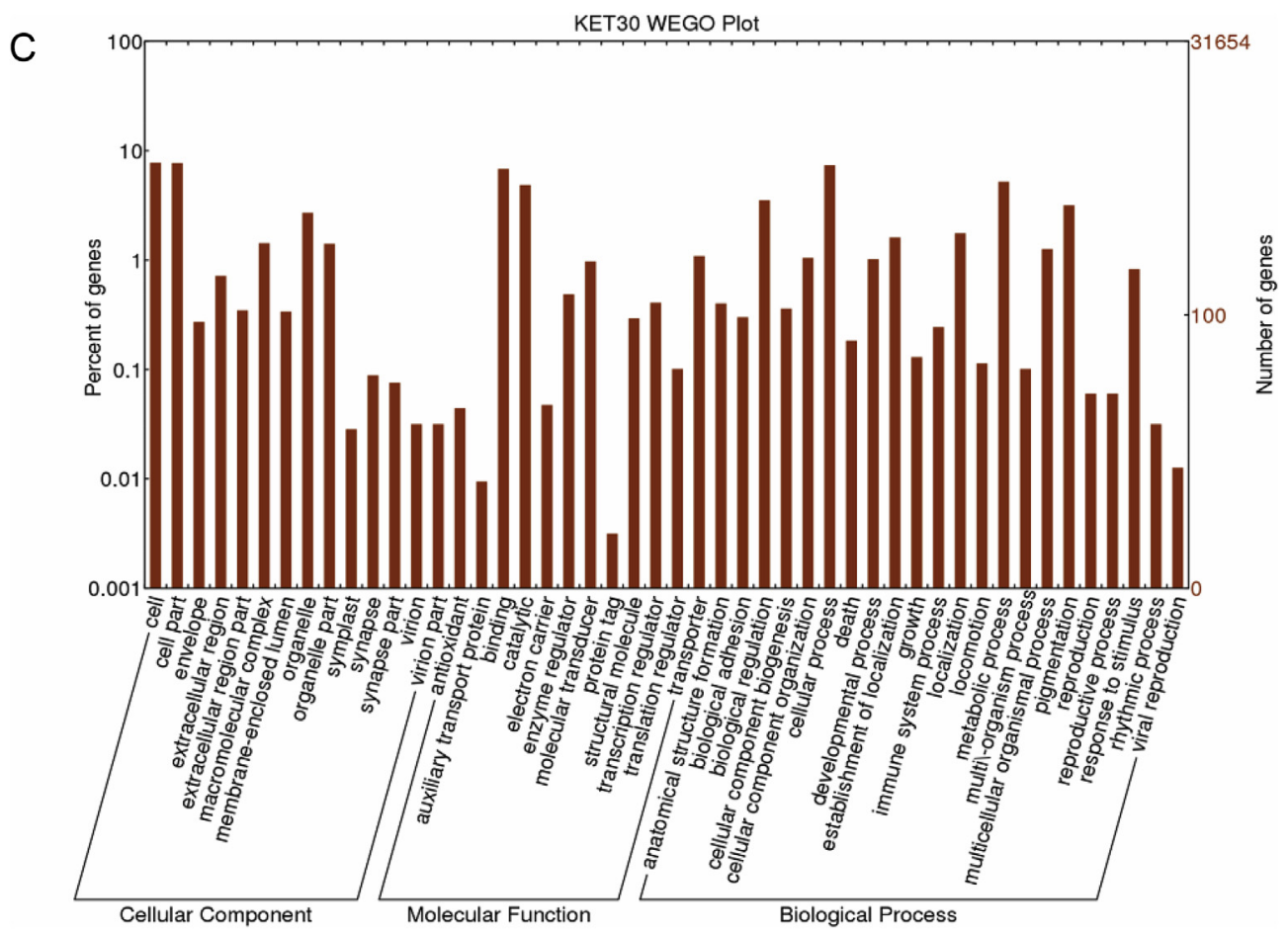

Fig. 1a. Top Hit Species distribution in E. tetradactylum (Sample-KET ${ }_{25}$ ).

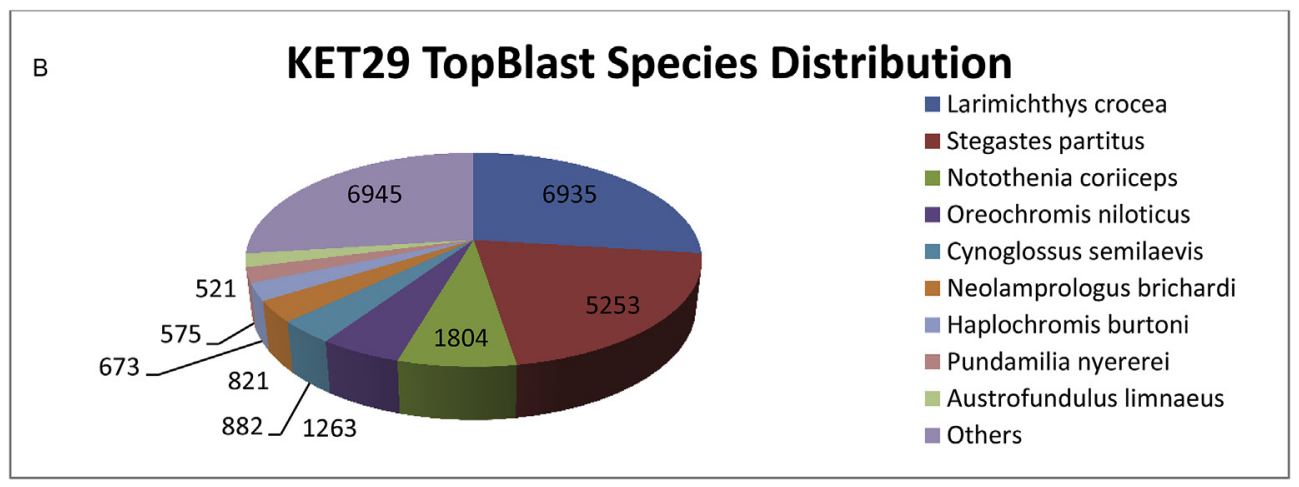

Fig. 1b. Top Hit Species distribution in E. tetradactylum (Sample-KET ${ }_{29}$ ).

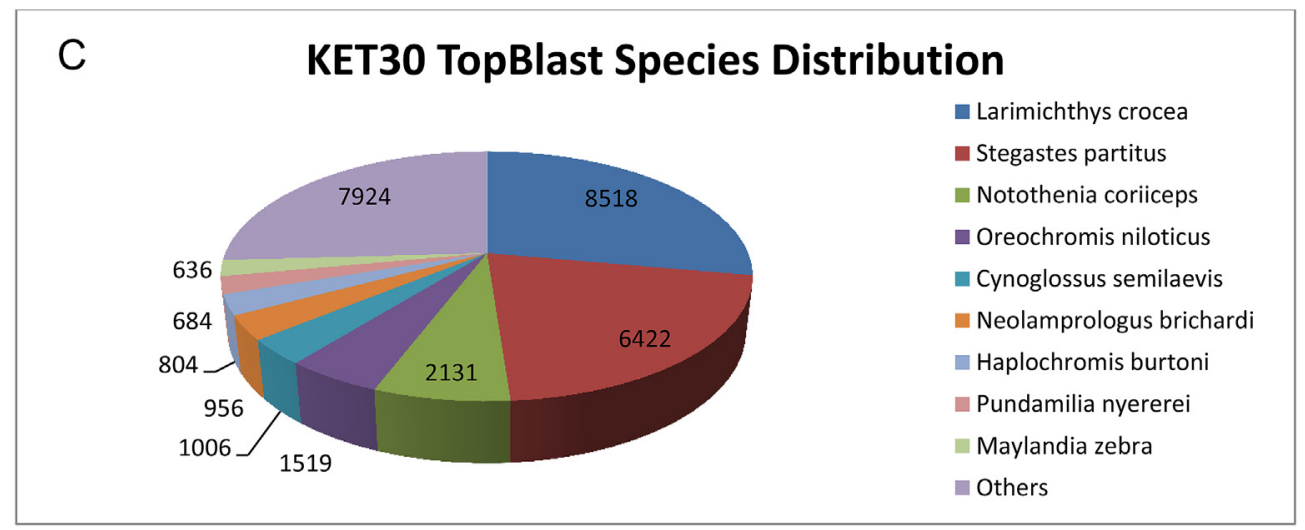

Fig. 1c. Top Hit Species distribution in E. tetradactylum (Sample-KET $\mathrm{K}_{30}$ ).

\subsection{De novo assembly and scaffolding}

The filtered high quality reads of the $\mathrm{KET}_{25}, \mathrm{KET}_{29}$ and $\mathrm{KET}_{30}$ samples were assembled in to scaffolds using CLCGENOMICS workbench v.9.5. The pre-assembled scaffolds were further assembled in to scaffold using SSPACE v.3.0 (SSAKE-based scaffolding of pre-assembled contigs after extension). 
Table 3

Gene Annotation Distributions in three samples of E. tetradactylum.

\begin{tabular}{lllll}
\hline Sr. No & $\begin{array}{l}\text { Sample } \\
\text { Name }\end{array}$ & $\begin{array}{l}\text { No. of } \\
\text { genes }\end{array}$ & $\begin{array}{l}\text { No. of genes with } \\
\text { Blast Hit }\end{array}$ & $\begin{array}{l}\text { No. of genes without } \\
\text { Blast Hit }\end{array}$ \\
\hline 1 & KET25 & 31,943 & 30,209 & 1734 \\
2 & KET29 & 26,487 & 25,107 & 1380 \\
3 & KET30 & 31,654 & 29,943 & 1711 \\
\hline
\end{tabular}

Table 4

Summary of Gene Ontology annotations in three samples of E. tetradactylum.

\begin{tabular}{lllll}
\hline Sr. No & $\begin{array}{l}\text { Sample } \\
\text { Name }\end{array}$ & $\begin{array}{l}\text { Biological } \\
\text { Processes }\end{array}$ & $\begin{array}{l}\text { Molecular } \\
\text { Functions }\end{array}$ & $\begin{array}{l}\text { Cellular } \\
\text { Component }\end{array}$ \\
\hline 1 & KET25 & 2980 & 3223 & 2459 \\
2 & KET29 & 2633 & 2866 & 2264 \\
3 & KET30 & 3054 & 3355 & 2647 \\
\hline
\end{tabular}

\subsection{Gene prediction}

AUGUSTUS (V.3.2.2) was used to predict genes from the assembled scaffolds with default parameters and Danio rerio was used as the model species.

\subsection{Functional annotation of genes and gene ontology}

The predicted genes of samples $\mathrm{KET}_{25}, \mathrm{KET}_{29}$ and $\mathrm{KET}_{30}$ were searched against NCBI non redundant protein (Nr) database using basic local alignment search tool (BlastX). Gene ontology (GO) annotations of the genes were determined by the Blast2GO programs. GO mapping was carried out in order to retrieve GO terms for all the BlastX functionally annotated genes. BlastX result accession IDs are used to retrieve gene names or symbols, identified gene name or symbols are then searched in the species specific entries of the gene-product tables of GO database.

\subsection{Simple sequence repeat (SSR) and polymorphic SSR identification}

The potential SSRs from three individual assemblies $\left(\mathrm{KET}_{25} ; \mathrm{KET}_{29}\right.$ and $\mathrm{KET}_{30}$ ) were identified as ranging from dinucleotide motifs with a minimum of ten repeats, tri nucleotide motif with minimum of three repeats, tetra, penta and hexa nucleotide motifs with a minimum of five repeats. A maximum distance of 200 nucleotides was allowed between two SSRs.

The scaffolds from three individual assemblies (KET25_Scaffold.fa, KET29_Scaffold.fa and KET30_Scaffold.fa) were clustered using CD-HIT $\mathrm{v} 4$ to generate a comprehensive reference. SSR prediction was done from the clustered reference assembly using MIcroSAtellite Identification Tool (MISA v1.0). Then we used PSR_read_retrieval script for identification of Polymorphic SSR Count based on reads mapping to the SSR region. The consensus sequence was called for each individual samples using high quality reads of $\mathrm{KET}_{25} ; \mathrm{KET}_{29}$ and $\mathrm{KET}_{30}$ mapped on reference assembly.

\section{Results and discussion}

Whole genome sequencing was performed on three samples of Indian salmon (E. tetradactylum) $\left(\mathrm{KET}_{25}, \mathrm{KET}_{29}\right.$ and $\left.\mathrm{KET}_{30}\right)$ using illumina NextSeq500 platform using $2 \times 150$ bp chemistry. 8,390,317, $7,085,775$ and $8,461,589$ high quality reads were obtained after trimming low quality reads and adapter sequence. These high quality reads obtained after the trimming were used for de novo assembly and obtained 289,461;280,260 and 289,650 scaffolds for $\mathrm{KET}_{25}, \mathrm{KET}_{29}$ and $\mathrm{KET}_{30}$ samples respectively. The detailed assembly statistics are provided in Table 1 . The gene prediction on assembled scaffolds predicted 31,943 genes for $\mathrm{KET}_{25} ; 26,487$ genes for $\mathrm{KET}_{29}$ and 31,654 genes for $\mathrm{KET}_{30}$ with average gene size of 458bp, 424bp and 459bp respectively. Predicted gene statistics are provided in Table 2.

A total of 30,209, 25,107 and 29,943 genes were annotated against the NCBI Nr database for the samples respectively. The majority of sequence similarity hits were found to be against the Larimichthys crocea (large yellow croaker) followed by Stegastes partitus (bicolor damselfish) for $\mathrm{KET}_{25}, \mathrm{KET}_{29}$ and $\mathrm{KET}_{30}$ samples (Fig. 1a, b \& c). Gene annotation distribution statistics are provided in Table 3. From the gene ontology (GO) analysis, 4,068, 3616 and 4182 genes were annotated for the given samples respectively. Summary of gene ontology annotations are provided in Table 4. GO assignments were used to classify the functions of the predicted genes. The GO mapping also provides ontology of defined terms representing gene product properties which are grouped in to three main domains such as biological process, molecular function and cellular component (Fig. 2a, b\& c). The GO category with the highest number of terms assigned biological processes, followed by cellular component while molecular functions had the least contigs

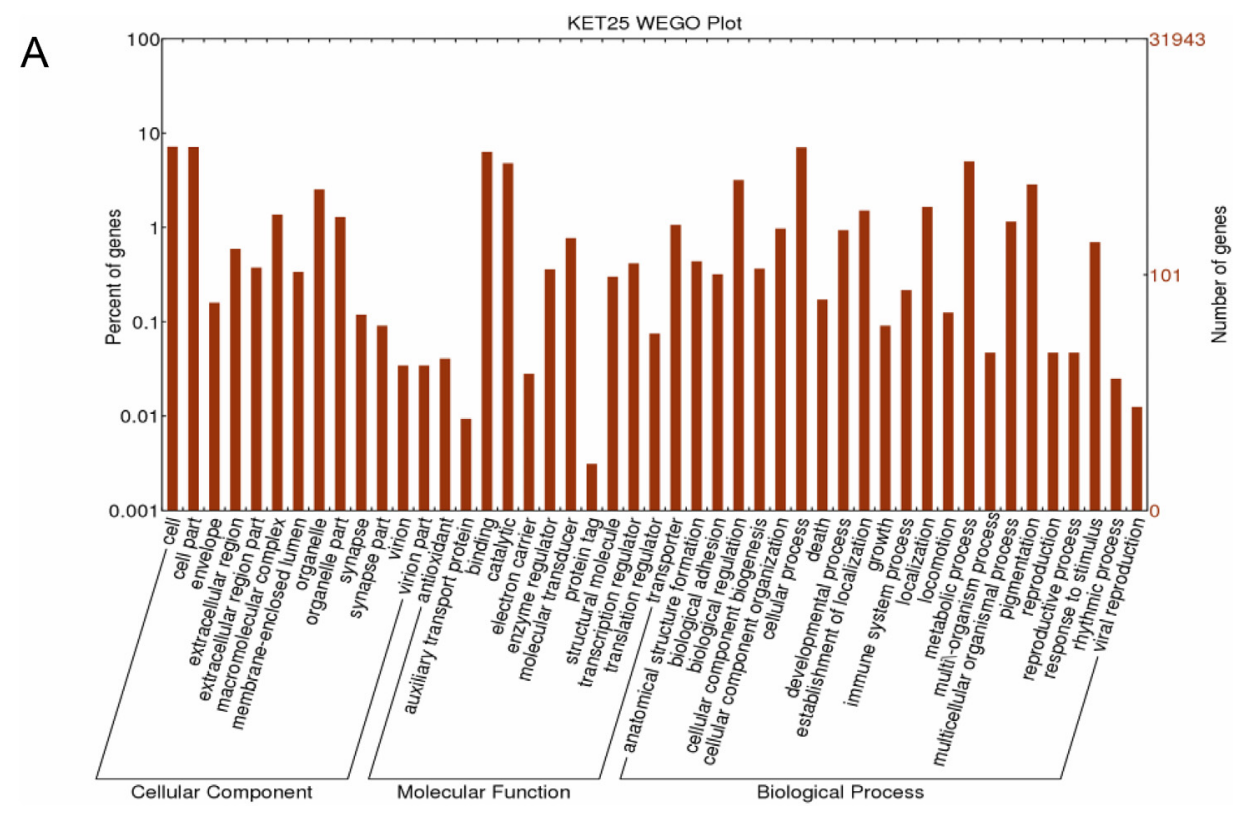

Fig. 2a. WEGO plot showing the Gene Ontology distribution in E. tetradactylum (sample - $\mathrm{KET}_{25}$ ). 


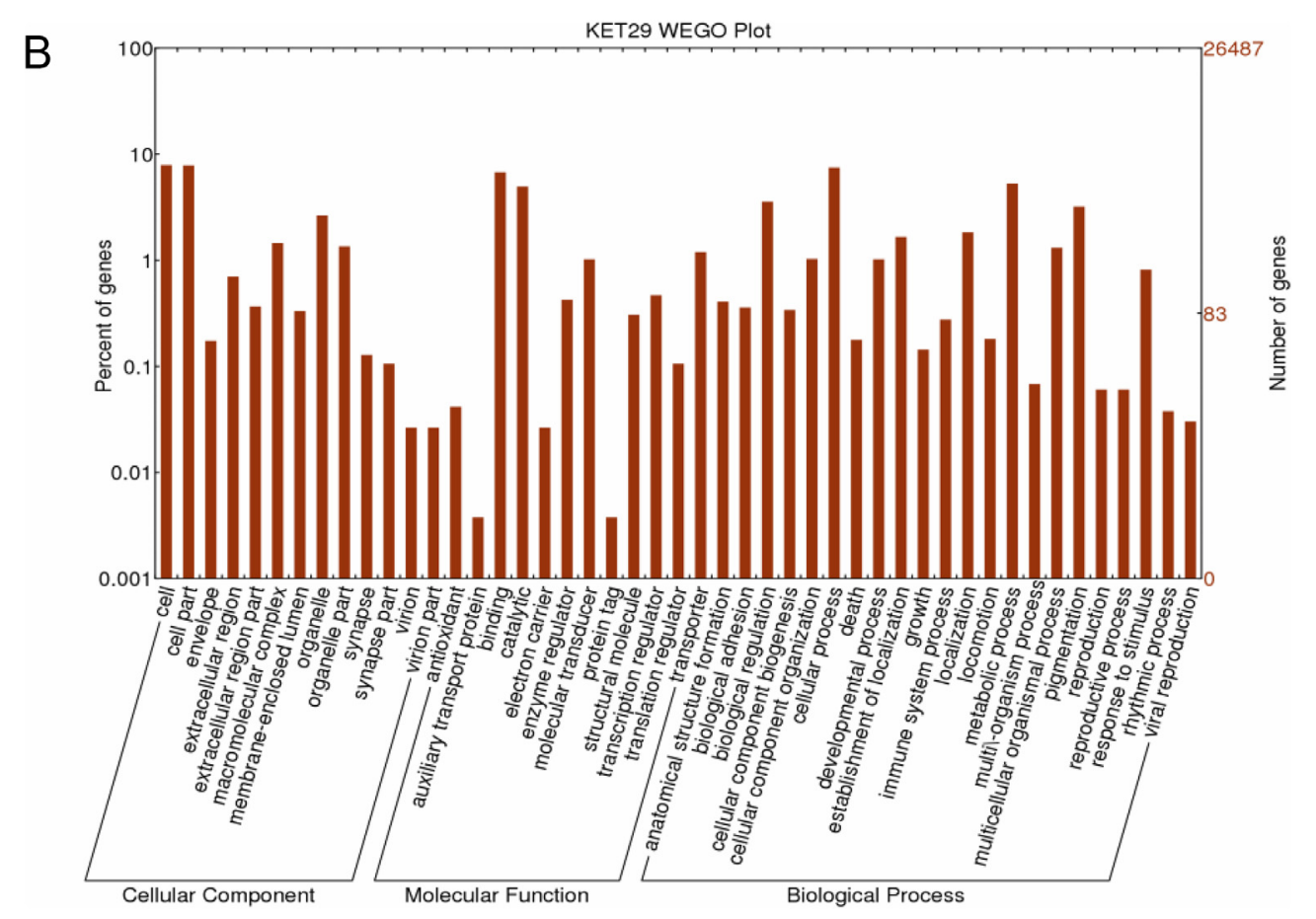

Fig. 2b. WEGO plot showing the Gene Ontology distribution in E. tetradactylum (sample- $\mathrm{KET}_{29}$ ).

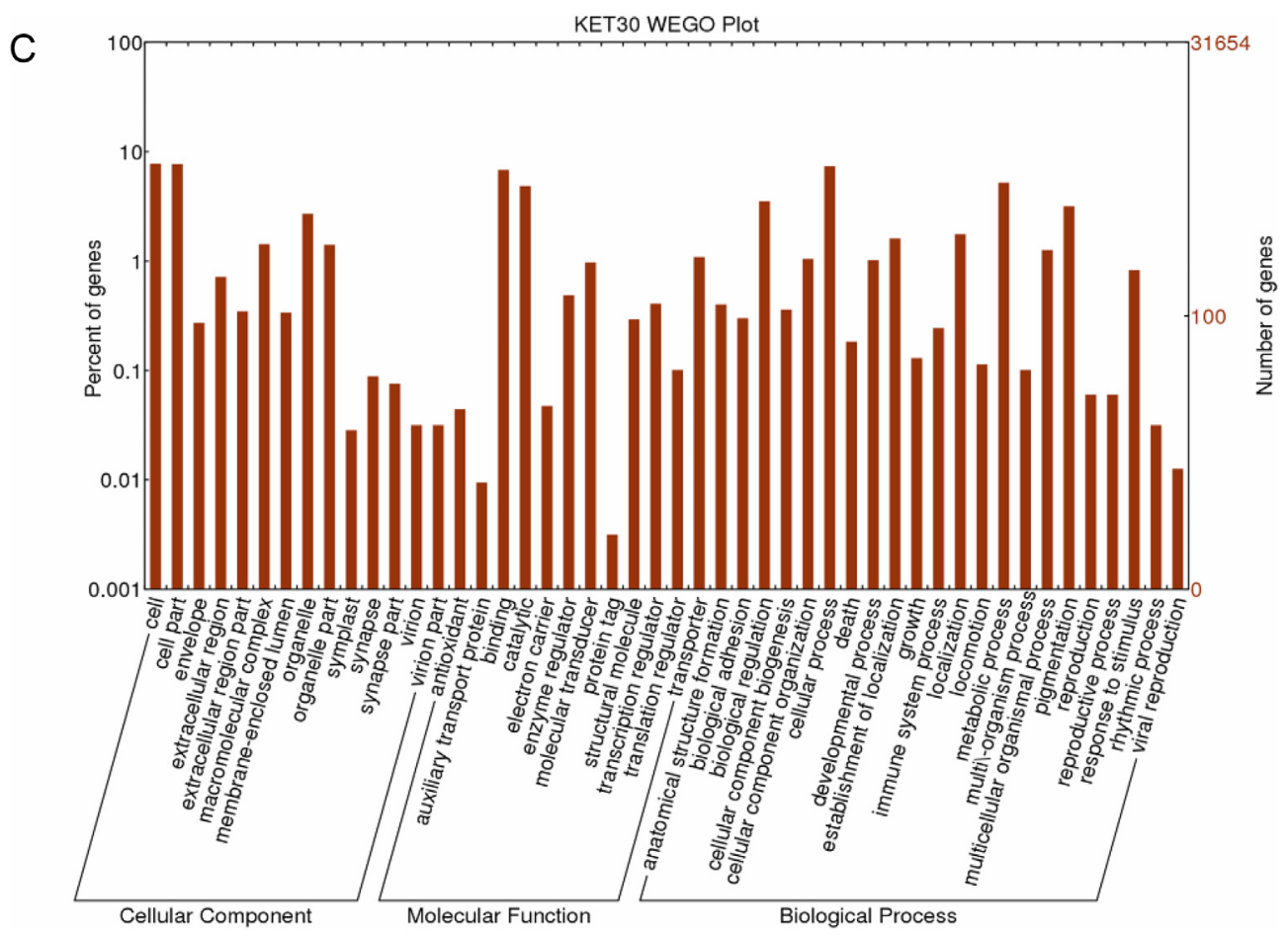

Fig. 2c. WEGO plot showing the Gene Ontology distribution in E. tetradactylum (sample-KET ${ }_{30}$ ).

assigned terms. The most commonly assigned GO terms in the biological processes GO category were the genes involved in cellular process and metabolic process. Intracellular and intrinsic components of membrane, were the most commonly assigned terms for the Cellular component GO category. The most commonly assigned GO terms for molecular function component were ion binding, catalytic and transporter respectively.

SSRs, also known as microsatellites, are tandem repeated motifs of 1-6 bases which are profusely dispensed across genomes and demonstrate high levels of allele polymorphism [11]. They serve as the most important molecular markers in population and conservation genetics, molecular epidemiology and pathology, and gene mapping. SSRs were detected using Microsatellite identification tool from assembled scaffolds. The potential SSRs were identified as ranging from dinucleotide motifs with a minimum of ten repeats, tri nucleotide motifs with minimum of three repeats, tetra, penta, and hexa nucleotide motifs with a minimum of five repeats. A maximum distance of 200 nucleotides was allowed between two SSRs. Out of 289,461 scaffold sequences examined in $\mathrm{KET}_{25}$ sample, 60,246 SSRs were identified. In $\mathrm{KET}_{29}$ sample, out of 280,260 scaffolds examined, 46,107 SSRs were identified. The 
Table 5

SSR Prediction statistics in three samples of E. tetradactylum.

\begin{tabular}{|c|c|c|c|}
\hline & KET25 & KET29 & KET30 \\
\hline Total number of scaffolds examined & 289,461 & 280,260 & 289,650 \\
\hline $\begin{array}{l}\text { Total size of examined sequences } \\
\text { (bp) }\end{array}$ & $301,704,044$ & $263,802,638$ & $306,253,351$ \\
\hline Total number of identified SSRs & 60,246 & 46,107 & 60,904 \\
\hline $\begin{array}{l}\text { Number of SSR containing } \\
\text { sequences }\end{array}$ & 48,664 & 38,543 & 49,191 \\
\hline $\begin{array}{l}\text { Number of sequences containing } \\
\text { more than } 1 \text { SSR }\end{array}$ & 8902 & 5950 & 9004 \\
\hline $\begin{array}{l}\text { Number of SSRs present in } \\
\text { compound formation }\end{array}$ & 5565 & 3918 & 5628 \\
\hline Di-nucleotide repeat & 15,430 & 9529 & 15,750 \\
\hline Tri-nucleotide repeat & 40,425 & 33,132 & 40,652 \\
\hline Tetra-nucleotide repeat & 3565 & 2846 & 3667 \\
\hline Penta-nucleotide repeat & 668 & 494 & 666 \\
\hline Hexa-nucleotide repeat & 158 & 106 & 169 \\
\hline
\end{tabular}

SSR prediction statistics are represented in Table 5 In $\mathrm{KET}_{30}$ sample, out of 289,650 scaffolds examined, 60,904 SSRs were identified. 23,983, 17,693 and 24,033 validated SSRs were obtained based on flanking sequence for $\mathrm{KET}_{25}, \mathrm{KET}_{29}$ and $\mathrm{KET}_{30}$ samples respectively. Based on common in silico validated SSR we have identified 1695 polymorphic SSR.

NGS methods can be used to address new and long-standing questions previously hindered by technological and financial limitations [2]. Microsatellite loci remain one of the most popular options for population genetic studies. The accessibility and throughput of NGS technologies has entitled the rapid and efficient microsatellite discovery by providing a greater amount of DNA sequencing reads at lower costs compared to other techniques (Irias et al., 2013). Massive and vast amounts of sequence data for a single or multiple individuals in a single run, low sequencing cost per base, reduction of the role of cloning and polymerase chain reaction (PCR) and, thus, reduced bias in resulting sequences; and the ability to identify rare variant sequences rather than a single sequence are the benefits of NGS technology when compared to the conventional capillary based sequencing [2]. NGS tools are also valuable for the discovery, validation and assessment of genetic markers in populations (Davey et al., 2011).

Accurate de novo assembly is critical for NGS projects in non-model organisms. The NGS and mining of the E. tetradactylum genome helped in identification of thousands of SSR markers. This vast sequenced data obtained by the de novo assembly and scaffolding can be used for developing polymorphic microsatellite markers and which will be useful in population genetic analysis and genotyping and conservation strategies in Indian salmon. The functional gene annotations and gene ontology results are useful for further gene expression studies in $E$. tetradactylum.

Here, we provide a gateway for the fishery biologist by providing NGS data to make such methods more broadly applicable and potential applications in several subfields of fishery biology. The vast data generated here in this study through NGS technology will be useful for the microsatellite marker development, gene expression studies, gene mining for novel genes and other related studies in this low volume high value species.

\section{Conflicts of interest}

The authors declare that they have no competing interests.

\section{Data availability}

The raw sequencing reads were deposited to the NCBI Sequence
Read Archive as part of Bioproject PRJNA450893. The Illumina NextSeq500 reads are available through the NCBI SRA accession number SRP141102.

\section{Acknowledgments}

The authors deeply thank Indian Council of Agricultural Research for providing the fund and deeply acknowledge the support and help of Dr. Prathibha Rohit, Mohammed Koya, Ms Muktha Menon, Dr. Akhilesh KV, Dr. MP Paulton, Dr Jeena NS. We thank Eurofins Genomics India Private limited (Bangalore, India) for the NGS library preparation and microsatellite identification.

\section{References}

[1] J.W. Davey, M.L. Blaxter, RADSeq: next-generation population genetics, Briefings Funct. Genomics 9 (2010) 416-423.

[2] H.R. Lerner, R.C. Fleischer, Prospects for the use of next-generation sequencing methods in ornithology, Auk 127 (2010) 4-15.

[3] E.R. Mardis, Next- generation sequencing platforms, Annu. Rev. Anal. Chem. 6 (2013) 287-303.

[4] T. Malausa, A. Gilles, E. Meglécz, H. Blanquart, S. Duthoy, C. Costedoat, N. Feau, et al., High-throughput microsatellite isolation through 454 GS-FLX Titanium pyrosequencing of enriched DNA libraries, Mol. Ecol. Res. 11 (2011) 638-644.

[5] L. Liu, Y. Li, S. Li, N. Hu, Y. He, R. Pong, D. Lin, L. Lu, M. Law, Comparison of nextgeneration sequencing systems, BioMed Res. Int. 5 (2012) 2012.

[6] T.N. Jennings, B.J. Knaus, T.D. Mullins, S.M. Haig, R.C. Cronn, Multiplexed microsatellite recovery using massively parallel sequencing, Mol. Ecol. Resour. 11 (2011) 1060-1067.

[7] S.R. Narum, C.A. Buerkle, J.W. Davey, M.R. Miller, P.A. Hohenlohe, Genotyping by sequencing in ecological and conservational genomics, Mol. Ecol. 22 (2013) 2841-2847.

[8] J. Wang, X. Yu, K. Zhao, Y. Zhang, J. Tong, Z. Peng, Microsatellite development for an endangered bream Megalobrama pellegrini (Teleostei, Cyprinidae) using $454 \mathrm{se}-$ quencing, Int. J. Mol. Sci. (2012) 3009-3021 2012 Mar 6.

[9] R.J. Haasl, B.A. Payseur, Multi-locus inference of population structure: a comparison between single nucleotide polymorphisms and microsatellites, Heredity 106 (2010) 158-171.

[10] L. Hauser, J.E. Seeb, Advances in molecular technology and their impact on fisheries genetics, Fish Fish. 9 (2008) 473-486.

[11] D.A. Chistiakov, B. Hellemans, F.A. Volckaert, Microsatellites and Their Genomic Distribution, Evolution, Function and Applications: a Review with Special Reference to Fish Genetics, Aquaculture, 2006 2551-29.

[12] L. Zane, L. Bargelloni, T. Patarnello, Strategies for microsatellite isolation: a review, Mol. Ecol. 11 (2002) 1-16.

[13] T.C. Glenn, N.A. Schable, Isolating microsatellite DNA loci, Methods Enzymol. 395 (2005) 202-222.

[14] A.I. Putman, I. Carbone, Challenges in analysis and interpretation of microsatellite data for population genetic studies, Ecol. Evol. 4 (2014) 4399-4428.

[15] B.J. Darby, S.F. Erickson, S.D. Hervey, S.N. Ellis- Felege, Digital fragment analysis of short tandem repeats by high-throughput amplicon sequencing, Ecol. Evol 6 (2016) 4502-4512.

[16] L. Zhan, I.G. Paterson, B.A. Fraser, B. Watson, I.R. Bradbury, P. Nadukkalam Ravindran, ... P. Bentzen, MEGASAT: automated inference of microsatellite genotypes from sequence data, Mol. Ecol. Resour. 17 (2) (2017) 247-256.

[17] I. Shihab, A. Gopalakrishnan, N. Vineesh, M. Muktha, K.V. Akhilesh, P. Vijayagopal, Histological profiling of gonads depicting protandrous hermaphroditism in Eleutheronema tetradactylum, J. Fish Biol. 90 (6) (2017) 2402-2411.

[18] H. Motomura, Threadfins of the World. An Annotated and Illustrated Catalaogue of Polynemid Species Known to Date. Family Polynemidae. FAO Species Catalogue for Fishery Purpose No. 3, Rome, (2004), p. 117.

[19] M.B. Pember, Characteristics of Fish Communities in Coastal Waters of NorthWestern Australia, Including the Biology of the Threadfin Species Eleutheronema tetradactylum and Polydactylus macrochir, Doctoral dissertation, Murdoch University, 2006.

[20] S.J. Newman, M.B. Pember, B.M. Rome, G.E. Mitsopoulos, C.L. Skepper, Q. Allsop, T. Saunders, A.C. Ballagh, L. Van Herwerden, R.N. Garrett, N.A. Gribble, Stock structure of blue threadfin Eleutheronema tetradactylum across northern Australia as inferred from stable isotopes in sagittal otolith carbonate, Fish. Manag. Ecol. 18 (2011) 246-257.

[21] M.T. Zischke, T.H. Cribb, D. Welch, W. Sawynok, R.J. Lester, Stock structure of blue threadfin Eleutheronema tetradactylum on the Queensland east coast, as determined by parasites and conventional tagging, J. Fish Biol. 75 (2009) 156-171.

[22] D.J. Welch, A. Ballagh, S.J. Newman, R.J. Lester, B. Moore, L. Van Herwerden, J. Horne, Q. Allsop, T. Saunders, J. Stapley, N.A. Gribble, Defining the Stock Structure of Northern Australia's Threadfin Salmon Species, (2010). 